\title{
CSR - zur Bürgerrolle und Verantwortung von Unternehmen
}

\author{
Thomas Beschorner und Christoph Schank
}

\section{Einleitung}

Die Konzepte Corporate Social Responsibility (CSR) und Corporate Citizenship (CC) zeichnen sich auch nach Jahren der wissenschaftlichen Auseinandersetzung und mehreren politischen Standardisierungsversuchen durch einen enormen Bedeutungspluralismus aus. Neben dem steigenden Bewusstsein dafür, dass Unternehmen gerade in einer globalisierten Weltgesellschaft nicht mehr nur als ökonomische Akteure zu begreifen sind, kann nicht zuletzt in den definitorischen Unschärfen der Begriffe ein Grund für die hohe Faszination der Konzepte erkannt werden. Schon längst fristen Corporate Social Responsibility und Corporate Citizenship kein Nischendasein mehr, sondern haben breiten Einzug in die Managementforschung und -literatur gefunden. ${ }^{1}$

Vielleicht ist jedoch der rasante Einzug der Begriffe in der Unternehmenspraxis selbst noch bemerkenswerter. Unternehmen verwenden die Begriffe - mal den einen, mal den anderen, manchmal beide - als Etikett für ihre gesellschaftliche Verantwortung oder ihr Selbstverständnis in der Gesellschaft. Gegenwärtig erscheinen beide Begriffe attraktiv, verheißungsvoll - und doch gleichzeitig von einem Dasein als Leerformel bedroht. Dabei sticht besonders eine Beliebigkeit oder zumindest Vieldeutigkeit in der Abgrenzung von Corporate Social Responsibility und Corporate Citizenship ins Auge. Nebeneinander finden sich hier in der wissenschaftlichen Literatur Gleichsetzungen wie auch Über- und Unterordnungen der Begriffe. Geeint werden beide Konzepte darin, gegenüber der Wirtschafts- und Unternehmensethik (vermeintlich) weniger moralisierend und akademisch daherzukommen. ${ }^{2}$ Darüber hinaus wird die Metapher vom Good Citizen, vom guten Bürger, gerne bemüht, um die Zweckorganisation Unternehmen als ,mit Kopf, Herz und Hand agierenden Menschen" ${ }^{\text {"3 }}$ zu personifizieren.

Als viel zitierte Quelle für die Verunsicherung in der definitorischen Abgrenzung zwischen Corporate Citizenship und Corporate Social Responsibility gilt Archie B. Carroll, der in seinem klassischen Aufsatz von 1991 die Unternehmen dazu auffordert, sich als Good Citizen zu etablieren. ${ }^{4}$ Er definiert Corporate Citizenship an dieser Stelle, ohne den Begriff explizit zu verwenden, als philanthropisches

\footnotetext{
Matten, D.; Palazzo, G. (2008); Hansen, U.; Schrader, U. (2005)

Göbel, E. (2006), S. 200

Schrader, U. (2011), S. 309

Carroll, A. B. (1991), siehe auch Carroll, A. B. (1979)
} 
und abschließendes Moment einer vierstufigen Pyramide der Corporate Social Responsibility. Während an dieser Stelle Corporate Citizenship noch als Teilaspekt der Corporate Social Responsibility verstanden wird, setzt Carroll selbst 1998 beide Begriffe gleich beziehungsweise löst er Corporate Social Responsibility durch Corporate Citizenship ab. ${ }^{5}$ Bereits durch die Gegenüberstellung dieser beiden Beiträge kann die bis heute nicht abschließend beantwortete Frage illustriert werden, ob sich unternehmerische Verantwortung allein im philanthropischen Engagement zeigt.

Wir wollen in diesem Beitrag dieses babylonische Sprach- und Bedeutungsgewirr mit dem Begriff „Corporate Citizen Responsibility“ (CCR) plakativ aufzulösen versuchen, indem wir die beiden Kernbegriffe von Corporate Citizenship und Corporate Social Responsibility, nämlich den Bürger- und den Verantwortungsbegriff, näher betrachten und miteinander in Beziehung setzen werden. Die Bürgerrolle des Unternehmens (Corporate Citizenship) wird dabei konzeptionell als ein gedachtes Ideal für eine Verhältnisbestimmung von Unternehmen zu ihren staatlichen wie zivilgesellschaftlichen Umwelten begriffen, dem sich über inkludierende Diskurse angenähert werden kann.

Für den Untersuchungszweck eruiert der folgende Abschnitt 2 in kurzer Form den Bürgerbegriff im Liberalismus und Republikanismus, aus dem dann im Abschnitt 3 ein angemessenes Corporate-Citizenship-Konzept entwickelt wird. Im Abschnitt 4 führen wir diese Überlegungen durch einen knappen Rekurs auf den Verantwortungsbegriff weiter. Wir schließen mit einem Fazit in Abschnitt 5.

\section{Bourgeois und Citoyen: Zum Bürgerbegriff im Liberalismus und Republikanismus}

Die Diskurse um Bürgerrechte und Bürgerpflichten finden in der abendländischen politischen Philosophie ihre Orientierungspunkte vornehmlich im Liberalismus und Republikanismus.

Das Bürgerbild des Liberalismus, wie es maßgeblich von Thomas Hobbes und John Locke begründet wurde, ist jenes des Besitzbürgers, des Bourgeois. ${ }^{6}$ Die liberale Tradition betont die Rechte des Bürgers, die es vor seinesgleichen ebenso wie vor einem übermächtigen Staat zu schützen gilt. Der Bürger ist hier ein Besitzbürger, da er zentrale Rechte an Leben, sichergestellt durch die öffentliche Ordnung, und Besitz hält. Der Bourgeois ist damit ein Akteur, dem in gemeinschaftlich gesteckten und über einen Staatsvertrag reglementierten Grenzen die vorrangige Verfolgung des eigenen Vorteiles ausdrücklich gestattet bleibt. Unter den Bürgerpflichten gilt der Rechtsgehorsam als die nobelste, mitunter auch die einzig relevante Pflicht. Übertragen auf die Unternehmen bedeutet dies, stets die Gesetze zu achten, in diesem Rahmen dann aber mit aller Kraft den eigenen Vorteil zu verfolgen.

Carroll, A. B. (1998)

vgl. auch Schrader, U. (2003) 
Die Anziehungskraft dieses Bürgerbegriffes erscheint für Unternehmen in vielfacher Hinsicht besonders ausgeprägt. Zum einen stehen sie somit in einer Tradition, die staatliche Angriffe auf die Freiheitsgrade ihrer Unternehmensführung abwehrt und ihnen durch die rein philanthropisch orientierte Übernahme von Verantwortung präventiv vorbeugt, zum anderen wird das Recht auf unbedingte Gewinnerzielung und freie Gewinnverwendung betont. Daran besonders anschlussfähig sind solche Ansätze von gesellschaftlicher Verantwortung, die in der Erzielung und Verwendung der Gewinne getrennte Bereiche erkennen, die unabhängig voneinander zu verfolgen sind. Eine derartige karitativ verstandene Unternehmensethik kann Unternehmen dazu veranlassen, legitime gesellschaftliche Ansprüche an die moralische Integrität mit einem dem Kerngeschäft nachgelagerten Engagement für das Gemeinwesen zu begegnen. Für eine das Gemeinwohl befördernde bürgerschaftliche Verantwortung durch Unternehmen befähigt der liberalistische Ansatz in seiner reinen Form insofern kaum, da ein Zusammenwirken von Engagement und Kerngeschäft nicht eingefordert wird. ${ }^{7}$

Dem Besitzbürger des Liberalismus steht im Republikanismus der Staatsbürger, der Citoyen, gegenüber. Die entscheidend von Aristoteles und Rousseau geprägte republikanische Tradition übt auf die deutschsprachige Wirtschaftsethik großen Einfluss aus, wie die Ansätze von Peter Ulrich sowie Horst Steinmann und Albert Löhr zeigen. Der Staatsbürger ist nun ein Akteur, der seinen Sonderwillen nicht über das Gemeinwohl stellt, sondern für den die Förderung des gemeinsamen Nutzens der Gesellschaft selbst das höchste Gut ist. Seine Bürgerschaft begründet sich nicht durch seine negative Freiheit, das heißt die Abwehr von Schaden, Unfreiheit und Entrechtung, sondern durch seine freiwillig übernommenen Verpflichtungen gegenüber dem Gemeinwesen. Während das Kondensat des guten Bürgers im Liberalismus noch allein der Rechtsgehorsam darstellt, misst sich gutes Bürgertum im Republikanismus durch den Beitrag zum Gemeinwesen. So wie der Liberalismus sich, bei aller erkannter Notwendigkeit für eine ordnende und individuelle Rechte schützende Rahmenordnung, gegenüber dem Staat kritisch eingestellt zeigt, so sehr definiert sich der republikanische Staatsbürger über seine politische Partizipation am Herrschen und beherrscht werden. ${ }^{8}$

Diese politische Orientierung am Gemeinwohl gilt ausdrücklich auch in der Sphäre des Marktes; gute Bürger zeichnen sich gerade durch die gemeinsame Betrachtung von Bürgersinn und Geschäftssinn aus. ${ }^{9}$ Ein republikanischer Bürger stellt damit eine untrennbare Einheit zwischen dem am Eigenwohl orientierten und dem Gemeinwohl verpflichteten Akteur dar. Dadurch steht Wirtschaften stets unter einem Legitimitätsvorbehalt und unterliegt einer gesellschaftlichen Aushandlung. Diese Rechtfertigung gilt, wie die bisherigen Ausführungen vermuten lassen könnten, jedoch nicht allein dem Staat als Verkörperung des Gemeinwesens. Gerade der Kommunitarismus rückt die Beziehungen zwischen den Bürgern selbst stärker in den Vordergrund und erkennt in bürgerschaftlichem Engagement ein

\footnotetext{
Moon et al. (2003). S. 7 f.

Aristoteles (1994), S. 135

9 Ulrich, P. (2005), S. 14
} 
Mittel zur Überwindung regulativer Defizite der staatlichen Rahmenordnung, wie sie unter dem Eindruck einer zunehmend globalisierten Weltgesellschaft und Wirtschaftsordnung deutlich zutage treten. ${ }^{10}$ Ein guter Bürger im Republikanismus zeichnet sich daher nicht durch eine Verteidigung seiner persönlichen Freiheitsrechte nach Außen aus, sondern gewinnt seine Qualität durch die unbedingte Rückkoppelung seines eigenen Vorwärtsstrebens an das Gemeinwohl. Ein von den Belangen und Ansprüchen Dritter unbeeinflusstes Wirken ist ihm nicht möglich. Aus diesen Wesensmerkmalen des republikanischen Bürgers resultiert seine hohe Eignung für ein wohlverstandenes gutes Unternehmensbürgertum, wie es später noch aufzuzeigen gilt.

\section{Der Corporate Citoyen}

Über die Theoriedifferenzen beider Bürgerschaftstheorien hinweg, eint beide Ansätze ein Bürgerverständnis, das gerade für den Corporate Citizen wesentlich ist und eines daran ausgerichteten Diskurses bedarf. Ein korporativ verfasster Bürger, das heisst eine auf einen Organisationszweck ausgerichtete Vereinigung von Individuen als Trägerin sui generis von bürgerlichen Rechten und Pflichten, existiert in der politischen Philosophie schlichtweg nicht.

Die Zuerkennung eines Bürgerstatus für Unternehmen ist damit keinesfalls voraussetzungslos. Eine Annäherung an diese Problematik kann über die Organisationstheorie gelingen, wie sie gerade in und für die Wirtschaftswissenschaften zu essenziellen Theoriemomenten geführt hat. Als kleinste Bausteine einer Organisation gelten die in ihr handelnden oder zumindest ihr zugerechneten natürlichen Personen sowie in ihr wirkenden Institutionen. ${ }^{11}$ Die organisationalen Institutionen dienen der Verfolgung des Organisationszweckes, der für die Entstehung der Organisation überhaupt erst ursächlich ist. Durch die klare Zuordnung der natürlichen Personen, des verbindenden und verbindlichen Institutionen-Sets (einschließlich eines formaljuristischen Gründungsaktes) wird eine dauerhafte Grenzziehung zwischen Unternehmen und ihrer Umwelt erlaubt, wie sie für natürliche Personen selbstverständlich ist. Für artifizielle Gebilde gilt diese Selbstverständlichkeit keineswegs und folglich ist die Unterscheidbarkeit zwischen Mitgliedern und Nicht-Mitgliedern, Innen und Außen, ein zentrales Merkmal einer autonom agierenden und stabilen Organisation, wie sie Unternehmen klassischerweise darstellen. ${ }^{12}$

Unter diesen Erwägungen ist die Rede vom korporativen Bürger nicht nur möglich, sondern es ist auch folgerichtig, Unternehmen als kollektive Akteure zu verstehen. Unternehmen verfolgen ,in einem formalen, legitimierten Prozess" ${ }^{\text {"13 }}$ formulierte Zielsetzungen, die durch gemeinsame Handlungen erreicht werden

\footnotetext{
${ }^{10}$ Schrader, U. (2011), S. 308

${ }^{11}$ North, D. C. (1992), S. 5

${ }_{12}$ Schreyögg, G. (1999), S. 9

${ }^{13}$ Kieser, A.; Walgenbach, P. (2003), S. 8
} 
sollen. Sie bleiben dabei als Summe ihrer Teile nicht diffus, sondern sind klar von der sie umgebenden Umwelt abgegrenzte Akteure, denen Handlungen und Handlungsfolgen zugerechnet werden können.

Diesen Befund formuliert Schrader ${ }^{14}$ in eine Forderung um: Damit Unternehmen als gute Bürger fungieren können, müssen sie eine konsistente Unternehmensidentität ausbilden. Diese auszubildende Identität erschöpft sich nicht bereits in der Abgrenzung zur Umwelt, sondern fordert ein in sich stimmiges Gesamtbild der Unternehmung ein. Dies impliziert, dass Kerngeschäft und philanthropische Aktivitäten keine voneinander losgelösten Bereiche darstellen. Corporate Citzenship ist nicht auf die dem Kerngeschäft nachgelagerte Gewinnverwendung beschränkt. Es geht nicht darum, wie Unternehmen ihre Gewinne verwenden, sondern wie sie diese erwirtschaften.

Wir können damit auch festhalten, dass es insbesondere mit einer republikanischen Bürgerschaftstheorie möglich wird, Unternehmen den gesellschaftlichen Status als korporative Bürger zu eröffnen. Dies gelingt über die freiwillig verfolgte Bestrebung zur Mehrung des Gemeinwohles durch die Übernahme gesellschaftlicher Verpflichtungen. Eine vollständige Gleichsetzung des natürlichen mit dem korporativen Bürgers ist dazu keinesfalls notwendig, wie auch Moon, Matten und Crane betonen: „corporations could reasonably claim to act as if they were metaphorically citizens in that their engagement in society resembles the key process of citizenship, participation."15

Das Mitwirken am Politischen, die „Teilhabe der Unternehmung an gesellschaftlicher Governance" ${ }^{\text {"16 }}$, und nicht der wie auch immer materiell bestimmte Bürgerschaftsstatus, ist dabei die entscheidende Offerte an Unternehmen, sich in einem republikanischen Sinne als Corporate Citoyen zu begreifen und gesellschaftlich zu positionieren. Dies beinhaltet im Kern die Forderung, nicht allein vom Staat legitime Rechte in Anspruch zu nehmen, sondern auch zur Gestaltung eines funktionierenden Gemeinwesens freiwillige Verpflichtungen zu übernehmen. ${ }^{17}$ Die Anforderungen an Unternehmen lassen sich daher nicht auf die Dimensionen ökonomischer Tragfähigkeit und juristischer Rechtmäßigkeit des Handelns verengen, sondern beinhalten auch ausdrücklich die Forderung, die Partikularinteressen (volonté particuliére) der Gewinnerzielung mit der Gemeinwohlorientierung (volonté générale) in Einklang zu bringen. Gerade dies kann nicht über eine karitative Spendenethik, wie Corporate Citizenship mitunter fehlgedeutet wird, gelingen.

Ein normativ gehaltvolles Verständnis vom guten Unternehmerbürgertum in einer republikanischen Tradition stellt für die Praxis der Unternehmensführung eine enorme, vermutlich niemals vollständig einzulösende Entwicklungsaufgabe dar. Gutes Unternehmertum verlangt von Unternehmen, ihr Engagement für die Gesellschaft nicht nur in nachgelagerten, philanthropischen Aktivitäten zu manifestieren, sondern ihre vollständige Geschäftstätigkeit - und damit auch und ge-

\footnotetext{
${ }^{14}$ Schrader, U. (2011), S. 309; Schrader, U. (2006), S. 226

${ }^{15}$ Moon, J. et al. (2003), S. $20 \mathrm{f}$.

${ }^{16}$ Pfriem, R. (2004), S. 190

${ }^{17}$ vgl. auch Marsden, C. (2000), S. 11
} 
rade die Gewinnerzielung - unter das Primat einer Gemeinwohlorientierung zu stellen. Die Verfolgung von ökonomischen Eigeninteressen ist nur in dem Maße legitim, wie sie nicht dem gesellschaftlichen Interesse entgegensteht.

Die Spielregeln der Rahmenordnung bilden für Unternehmen hier eine erste, keinesfalls aber hinreichende Orientierungshilfe. Zugleich befreit ein korporatives Bürgertum die Unternehmen aus einer diskursiven Defensive. Als kollektive, aber womöglich dennoch vollwertige Bürger sind sie nicht länger allein Adressaten von Ansprüchen und Anforderungen, sondern ausdrücklich zur Mitgestaltung des Politischen aufgerufen. Dies führt zwangsläufig zu einer Neubewertung von Organisationszweck und organisationaler Handlungslogik. Die Ausschöpfung jeglicher legitimer Gewinnmöglichkeit steht dann unter dem Vorbehalt einer staatsbürgerlichen Verpflichtung. Von dieser Betrachtungsweise her sind verschiedene Weiterführungen möglich:

Erstens deutet sich an, dass die im deutschsprachigen Raum übliche Verwendung des Begriffs Corporate Citzenship als Corporate Giving und Corporate Volunteering eine verkürzte Sichtweise des Bürgerbegriffs ist: Corporate Giving stellt dabei in verschiedenen Formen von Sach- und Geldspenden ausschließlich auf den Bürger als Bourgeois ab. Corporate Volunteering ${ }^{18}$ meint ein von den Organisationsmitgliedern erbrachtes gesellschaftliches Engagement in Form ihrer kreativen, schöpferischen, organisierenden oder körperlichen Arbeitskraft. Beschränkt sich dies nur auf den „Output“ und „Outcome“ von erbrachten Leistungen, so stellt Corporate Volunteering lediglich eine weitere Form des Corporate Giving dar (Zeitspende). Die entscheidende Kategorie für ein angemessenes Bürgerverständnis im obigen Sinne wäre hingegen der „Impact“ der jeweiligen Maßnahme - und zwar in doppelter Hinsicht:

- Als wahre Bürgerpartizipation (mit anderen Bürgern); nicht das ehrenamtliche Anstreichen eines Klassenzimmers durch Unternehmensmitarbeiter, sondern die Planung, Materialbeschaffung und das Anstreichen eines Klassenzimmers gemeinsam mit benachteiligten Jugendlichen ist die wichtige Übung, im besten Fall mit Lernprozessen auf beiden Seiten, sowie

- hinsichtlich der Rückkoppelungseffekte - durch die Erfahrungen und Erlebnisse der Mitarbeiter in anderen Handlungskontexten mit anderen Handlungslogiken - auf die und in der Unternehmensorganisation.

Das Corporate-Citizenship-Konzept im Sinne eines republikanischen Bürgerverständnisses scheint uns, zweitens, sehr gut als Ideal für eine Verhältnisbestimmung von Unternehmen zu ihren staatlichen wie zivilgesellschaftlichen Umwelten geeignet. Als strukturelle Schwäche betrachten wir jedoch seine Abstraktheit und Ferne von konkreten wirtschafts- und unternehmensethischen Problemstellungen. Wir sehen dies insbesondere in der Schwierigkeit begründet, den normativen Gehalt dieses Ideals interaktionstheoretisch zu konzeptualisieren, und wir sehen zugleich

\footnotetext{
${ }^{18}$ Mögliche Ausdifferenzierungen von Corporate Citizenship finden sich bei Weber, M. (2008), S. 44; Schrader, U. (2011), S. 304; Damm, D.; Lang, R. (2001); Dresewski, F. et al. (2004)
} 
eine Möglichkeit, dies über den Begriff der Verantwortung angemessen bearbeiten zu können, wie wir im Folgenden in gebotener Kürze andeuten wollen.

\section{Von der Bürgerpflicht zur Ver-Antwortung}

Es mag überraschen, dass der Begriff der Verantwortung ein philosophisch noch recht junger Terminus ist. Kurt Röttgers ${ }^{19}$ zeigt in einer aufschlussreichen Darstellung, dass der Verantwortungsbegriff bis ins 20. Jahrhundert hinein in verschiedenen philosophischen Fachwörterbüchern nicht aufgeführt war. Die Gründe dafür sieht er u.a. darin, dass „,der Pflichtbegriff im 20. Jahrhundert ins Zwielicht“ geraten war und er ergänzt: „Es ist zwar grundsätzlich die gleiche Struktur, die das Pflichtgebot und die Rhetorik der Verantwortungsübernahme für eine übergeordnete Ganzheit charakterisiert, aber Kants Vernunftmonismus hat in letztlich nicht überzeugender Weise unterstellt, dass es keine wirklichen, d.h. unlösbaren Pflichtenkonflikte geben könne" ${ }^{\text {"20 }}$. Hinzu kamen die Gründungen und Entwicklungen der Sozialwissenschaften (insbesondere die Soziologie) ${ }^{21}$ im 20. Jahrhundert, die stärker als philosophische Ansätze dieser Zeit ihr Augenmerk auf soziale Beziehungen und Interaktionen legten und damit dem Verantwortungsbegriff sicherlich Vorschub leisteten.

Verantwortung ist die Intensivierung einer „Antwort“, der wiederum eine Frage vorausgegangen sein muss. Jemand verantwortet sich für etwas gegenüber jemanden, so eine klassische Formel. ${ }^{22}$ Man spricht und interagiert miteinander. Ursprünglich war unter diesem Begriff ausschließlich ein ,zur Verantwortung gezogen werden“ gemeint, ,was nichts anderes heißen kann, als dass man dahin bewegt wird, wohin man freiwillig nicht ginge, in eine Befragungssituation, in der man etwas, oder im Extremfall sich, zu verantworten hat". ${ }^{23}$ Man spricht heute darüber hinaus oft davon, dass jemand - mehr oder weniger freiwillig Verantwortung übernimmt, was stärker moralisch und weniger rechtlich konnotiert ist. In beiden Varianten liegt das zu Verantwortende in der Regel in der Vergangenheit und man spricht in der Literatur daher auch von „retrospektive“, „ex post“" oder ,,vergangenheitsbezogene“" Verantwortung. ${ }^{24}$

Von diesen beiden Verantwortungstypen können wir einen stärker zukunftsbezogenen Verantwortungsbegriff unterscheiden, den wir hier als prospektive Verantwortung bezeichnen wollen. Er kommt nicht erst zum Tragen, wenn das Kind schon in den Brunnen gefallen ist, sondern ist fester Bestandteil einer zukunftsgerichteten Strategie von Akteuren, auch z. B. Unternehmen. Prospektive Verantwortung ist individuell (Verantwortungssubjekt, ,jemand verantwortet sich $^{\text {") }}$, immer auf etwas Konkretes bezogen (das Verantwortungsobjekt, ,für et-

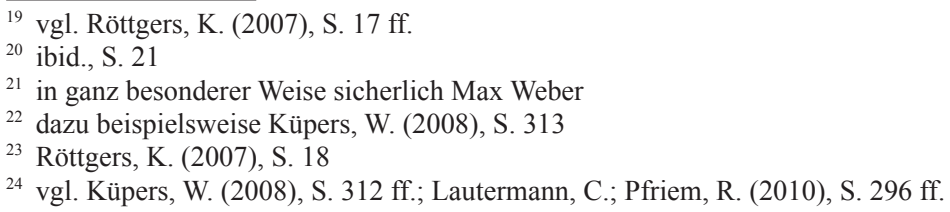


was"), richtet sich an Adressaten (die Verantwortungsinstanz, ,gegenüber jemanden") und orientiert sich dabei am Ideal eines republikanischen Bürgerbegriffs, also dem Bürger der „verantwortlich sein“ will und sich für diesen Zweck in die Gesellschaft einbringt.

Unsere Adjektivierung des Verantwortungsbegriffs in „verantwortlich sein“ deutet an, dass die Verantwortungsinhalte keinesfalls vorgegeben, sondern von den beteiligten oder betroffenen Akteuren prozessorientiert zu ermitteln und entwickeln sind. Wichtig ist in diesem Zusammenhang, erstens, dass dies immer in einem historisch-kulturellen Kontext stattfindet und zweitens, die Inklusion von Anspruchsgruppen nicht auf der Grundlage machtstrategischer Erwägungen „verhandelt", sondern „auf Augenhöhe“ realisiert wird. Lautermann und Pfriem formulieren dazu ähnlich: „Wo ein situatives, problembezogenes Lösen von heterogenen, prinzipiell gleichwertigen Ansichten darüber, was gut oder gerecht sei, gefragt ist, dort hilft als ethisches Konzept am besten die immer temporär und konkret anzuwendende Relation von Verantwortung weiter". ${ }^{25}$ Verantwortungsinhalte bestimmen sich damit also in Abhängigkeit von gesellschaftlichen Problemen, den jeweiligen Verantwortungssubjekten und in zentraler Art und Weise durch und in einem Diskurs mit den Beteiligten und Betroffenen.

Diese Weiterführung des Verantwortungsbegriffs ist für ein angemessenes Verständnis von Corporate Social Responsibility von größter Bedeutung, denn es geht um die entscheidende Frage, wie Unternehmen als Akteure eines gesellschaftlichen Wandels mobilisiert werden können. Es geht primär weder um die Verteilung von Spendengeldern noch um die Vermeidung von „bad practices“, wie Korruption, Bilanzfälschungen u.v.m., sondern um die Möglichkeiten und Grenzen der Realisierung von „good practices“ durch Unternehmen. Für derartige Zukunftsfragen benötigen wir angemessene Konzepte, wie einen prospektiven Verantwortungsbegriff, mit denen die Fragen auch bearbeitbar sind.

\section{Fazit}

Die Diskussion um die gesellschaftliche Verantwortung von Unternehmen gewinnt in Wissenschaft und Unternehmenspraxis weiter an Resonanz und Dynamik. Dies ist bereits Grund genug, das Verhältnis zweier bestimmender Konzepte zueinander, der Corporate Social Responsibility und dem Corporate Citizenship, über- und weiterzudenken. Wir haben bereits aufgezeigt, wie gutes Unternehmensbürgertum als ein voraussetzungsvolles, forderndes Konzept zu begreifen ist, dessen vollständige Einlösung durch die Unternehmen einem gedachten Idealzustand entspricht. Diesen Idealzustand erblicken wir in einem Unternehmertum, das sich als integraler Bestandteil der Gesellschaft versteht und gesellschaftliche Ansprüche nicht abzuwehren, sondern selbstreflexiv, antizipativ und interagierend zu erfüllen versucht. Wird der eingeschlagene Weg eines republikanisch orientierten Unternehmensbürgertums konsequent weiterverfolgt, erwachsen den Unternehmen daraus aber nicht

${ }^{25}$ Lautermann, C.; Pfriem, R. (2010), S. 295 
allein dem Gemeinwohl dienende Verpflichtungen, sondern sie werden zudem mit dem Mandat zur proaktiven gesellschaftlichen Gestaltung ausgestattet.

Die Annäherung an das Idealbild vom guten Unternehmensbürgertum stellt eine enorme Entwicklungsaufgabe dar, die sich auf Unternehmen, die Zivilgesellschaft und die Politik gleichermaßen erstreckt. Im Verständnis einer prospektiven Verantwortung ist das interaktionistische Moment besonders $\mathrm{zu}$ betonen, das Träger und Adressaten von Verantwortung in Diskurse über konkrete Problemlagen zusammenführt. Corporate Social Responsibility, sofern nicht lediglich als defensiv ausgerichtetes Konzept zur Vermeidung negativer Aufmerksamkeit und Abwehr staatlicher Regulierung begriffen, birgt das Potenzial zur Gestaltung dieser Diskurse und kann dazu beitragen, Unternehmen als gute Bürger zu einem verantwortungsvollen, am Gemeinwohl ausgerichteten Selbstbild zu verhelfen.

\section{Literatur}

Aristoteles (1994): Politik. Reinbeck: Rowohlt.

Carroll, A. B. (1979): A three-dimensional conceptual model of corporate performance. In: Academy of Management Review, Vol. 4, Nr. 4, S. 497-505.

Carroll, A. B. (1991): The pyramid of corporate social responsibility: toward the moral management of organizational stakeholders. In: Business Horizons, Vol. 34, Nr. 4, S. 39-48.

Carroll, A. B. (1998): The four faces of corporate citizenship. In: Business and Society Review. Vol. 100, Nr.1, S. 1-7.

Damm, D.; Lang, R. (2001): Handbuch Unternehmenskooperation. Erfahrungen mit Corporate Citizenship in Deutschland. Bonn: Stiftung Mitarbeit.

Dresewski, F.; Kromminga, P.;von Mutius, B. (2004): Corporate Citizenship oder: Mit solcher Verantwortung gewinnen, in: Wieland, J. (Hrsg.): Handbuch Wertemanagement. Hamburg: Murmann, S. 489-526.

Göbel, E. (2006): Unternehmensethik. Stuttgart: Lucius \& Lucius.

Hansen, U.; Schrader, U. (2005): Corporate Social Responsibility als aktuelles Thema der Betriebswirtschaftslehre. In: DBW, Vol. 65, Nr. 4, S. 373-395.

Kieser, A.; Walgenbach, P. (2003): Organisation. Stuttgart: Schäffer-Poeschel.

Küpers, W. (2008): Perspektiven responsiver und integraler Ver-Antwortung in Organisationen und der Wirtschaft in: Heidbrink, L. (Hrsg.): Verantwortung in der Marktwirtschaft, Frankfurt a.M.: Campus-Verlag, S. 307-338.

Lautermann, C.; Pfriem, R. (2010): Corporate Social Responsibility in wirtschaftsethischen Perspektiven., in: Raupp, J.; Jarolimek, S.; Schultz, F. (Hrsg.): Handbuch Corporate Social Responsibility. Kommunikationswissenschaftliche Grundlagen und methodische Zugänge, Wiesbaden: VS-Verlag, S. 281-304.

Mardsen, C. (2000): The new corporate citizenship of big business: Part of the solution to sustainability. In: Business and Society Review, Vol. 105, Nr. 1, S. 9-25.

Matten, D.; Crane, A. (2005): Corporate Citizenship - toward an extended theoretical conceptualization. In: Academy of Management Review, Vol. 30, Nr. 1, S. 166-179.

Matten, D.; Palazzo, G. (2008): Unternehmensethik als Gegenstand betriebswirtschaftlicher Forschung und Lehre - Eine Bestandsaufnahme aus internationaler Perspektive. In: Zeitschrift für betriebswirtschaftliche Forschung, Sonderheft 58, S. 50-71. 
Moon, J.; Crane, A.; Matten, D. (2003): Can corporations be citizens? Corporate citizenship as a metaphor for business participations in society ( $2^{\text {nd }}$ edition). No. 13-2003 ICCSR Research Paper Series.

North, D. C. (1992): Institutionen, institutioneller Wandel und Wirtschaftsleistung. Tübingen: Mohr Siebeck.

Pfriem, R. (2004): Ein pluralistisches Feld von Governancekulturen. In: Wieland, J. (Hrsg.): Governanceethik im Diskurs. Marburg: Metropolis, S. 183-21.

Röttgers, K. (2007): Verantwortung nach der Moderne in sozialphilosophischer Perspektive, in: Beschorner, T.; Linnebach, P.; Pfriem, R.; Ulrich, G. (Hrsg.): Unternehmensverantwortung aus kulturalistischer Sicht, Marburg: Metropolis, S. 17-31.

Schäfer, C. K. (2009): Corporate Volunteering und professionelles Freiwilligen-Management. Eine organisationssoziologische Betrachtung. Wiesbaden: VS-Verlag.

Schrader, U. (2006): Corporate Citizenship - Eine Innovation? In: Pfriem, R.; Antes, R.; Fichter, K.; Müller, M.; Paech, N.; Seuring, S.; Siebenhüner, B. (Hrsg.): Innovationen für eine nachhaltige Entwicklung. Wiesbaden: DUV, S. 231-248.

Schrader, U. (2011): Corporate Citizenship. In: Aßländer, M. S. (Hrsg.): Handbuch Wirtschaftsethik. Stuttgart und Weimar: J. B. Metzler, S. 303-312.

Schreyögg, G. (1999): Organisation. Grundlagen moderner Organisationsgestaltung. Wiesbaden: Gabler.

Ulrich, P. (2005): Zivilisierte Marktwirtschaft: Eine wirtschaftsethische Orientierung. Bern: Haupt.

Weber, M. (2008): Corporate Social Responsibility: Konzeptionelle Gemeinsamkeiten und Unterschiede zur Nachhaltigkeits- und Corporate-Citizenship-Diskussion. In: Müller, M.; Schaltegger, S. (Hrsg.): Corporate Social Responsibility. Trend oder Modeerscheinung? München: oekom, S. 39-51. 